\title{
Developmental change in partition dependent resource allocation behavior
}

Katherine Williams, Alexandra Zax, Sheri Reichelson, Andrea L. Patalano, \& Hilary Barth

Wesleyan University

Accepted for publication, Memory \& Cognition

This copy: Preprint 1.0, February 2020

Word Count: 4790

Corresponding author: Hilary C. Barth, Department of Psychology, Wesleyan University, 207 High St, Middletown, CT 06459, hbarth@wesleyan.edu

Author Note: We thank the participating children and families; the Connecticut Science Center; and research assistants in the Wesleyan University Cognitive Development Lab and the Wesleyan University Reasoning and Decision Making Lab. This work benefited from NSF DRL1561214. 


\begin{abstract}
Partition dependence, the tendency to distribute choices differently based on the way options are grouped, has important implications for decision making. This phenomenon, observed in adults across a variety of contexts such as allocating resources or making selections from a menu of items, can bias decision makers toward some choices and away from others. Only one study to date (Reichelson, Zax, Patalano, \& Barth, 2019) has investigated the developmental trajectory of this phenomenon. In the current study we investigate children's and adults' susceptibility to partitioning effects in a child-friendly resource allocation task. In Experiment $1(N=80)$, adults distributed 12 food tokens to animals at the zoo. Based on previous findings that older children show weaker partition dependence in this task, we predicted that adults might exhibit reduced partition dependent behavior: they showed none. In Experiment $2(N=272)$, we used a less transparent task with only five food tokens, predicting that both adults and children (ages 3-10 years) would show partition dependence. Children, but not adults, made partition dependent resource allocations, with younger children exhibiting greater effects than older children. These experiments provide further evidence that children's decisions, like adults' (in other tasks), are influenced by the arbitrary partitioning of the available options. This work supports previous findings that younger children may be more susceptible to these effects, and maps developmental change in partition dependent behavior from early childhood to adulthood on this child-friendly partition dependence task.
\end{abstract}

Keywords: partition dependence, decision making, cognitive development, resource allocation 
Developmental change in partition dependent resource allocation behavior

Our decisions can be shaped by the way options are presented. One such influence on decision making comes from the partitioning of the options: the same set of options can elicit different choices when partitioned in different ways. One reason for these partitioning effects is a phenomenon called diversification bias (Read, Antonides, Van den Ouden, \& Trienekens, 2001; Read \& Loewenstein, 1995; Simonson, 1990): decision makers demonstrate greater variety seeking, often diversifying their choices across all available options identified. When individuals diversify their choices over arbitrary groupings of options, rather than over the individual options themselves, diversification bias can lead to a broad phenomenon known as partition dependence (Fox \& Rottenstreich, 2003). Partition dependence, the tendency to make different choices based on the way options are grouped, can strongly influence decisions (Fox, Ratner, \& Lieb, 2005).

For example, imagine choosing six snacks for a movie, with popcorn, peanuts, or candy available for purchase. In the absence of strong preferences, adults tend to diversify their choices when presented with situations like this one (i.e., selecting a variety of snack options), perhaps choosing two of each, rather than choosing all of one option. Alternatively, if the hypothetical shopper subjectively partitions the available snack options into "salty" and "sweet" categories, they are likely to diversify their selections across these two categories rather than across the individual options, perhaps selecting half of their total snacks from the "salty" category and the other half from the "sweet" category. Partitioning in this way would lead to more individual candy selections and fewer popcorn and peanut selections.

Partition dependence has been observed in numerous adult consumer choice and allocation paradigms including snack selection, hypothetical wine selection, hypothetical allocations of money to financial aid or charities, and allocations of experiences to future time periods (Fox, Bardolet, \& Lieb, 2005; Fox, Ratner, et al., 2005; Simonson, 1990; Tannenbaum et al., 2015). For example, in one striking vignette-based study, the prescribing behavior of healthcare providers selecting one antibiotic treatment from an array of options was influenced by the partitioning of the available options. Participants were randomly assigned to select a treatment from a menu of options organized into one of two partition structures: over-the-counter options listed individually and prescription options grouped into a single row of response options, or the reverse menu grouping (Tannenbaum et al., 2015). Primary care providers chose aggressive treatments significantly more often when they were listed individually, compared to when they were grouped together. In another consumer-choice study, adults' hypothetical wine selections showed strong partitioning effects such that when wine descriptions were grouped by country of origin, participants diversified their selections over country of origin, but when descriptions were grouped by type of grape, they diversified their selections over type of grape instead (Fox, Ratner, et al., 2005)

Beyond cases of partition dependence in consumer choice, the partitioning of options can elicit different decisions in the context of allocating resources (e.g., time or money) over available options depending on how the options are subjectively grouped (Benartzi \& Thaler, 2001; Fox, Ratner, et al., 2005). For example, Fox, Bardolet, et al. (2005) asked graduate students to allocate limited financial aid resources to recipients in different household incomes and found that financial aid allocations were influenced by the partitioning of income brackets. In general, allocations reflected a need-based allocation criterion, but participants were also strongly biased by the income bracket partition they encountered. That is, while participants allocated more financial aid to lower income brackets overall, twice as much aid was allocated to 
families with incomes less than or equal to $\$ 75,000$ when this income bracket was partitioned into multiple brackets ( $96 \%$ of aid) compared to when it was presented as a single income bracket (48\%). This suggests that even when decision makers rely on need-based allocation criteria, they are also heavily influenced by the partitioning of options presented at the time a decision is made. This finding has since been replicated with a procedure that emphasized the arbitrariness of the partition structure, and strong partitioning effects were found even when both possible income partitions were explicitly presented to participants (Xing et al., in revision).

Some researchers have suggested that partition dependence results from a cognitive bias towards even allocation over all possibilities that have been identified, also more generally referred to as invoking maximum entropy heuristics (see Fox, Bardolet, et al., 2005 for discussion). For example, in decision contexts including consumer choice (i.e., variety-seeking) or resource allocation (i.e., naïve diversification), decision makers might first subjectively partition the set of options into groups (e.g., household incomes) and then distribute resources (e.g., financial aid resources) across all available options, sometimes with little regard to any distinguishing features of the available options. Thus, the way in which a set of options is described or presented at the time of a decision can strongly influence the partition the decision maker subjectively invokes, therefore altering their pattern of responses (Fox, Bardolet, et al., 2005). Such subjective partitioning can be naturally occurring in the choice environment, spontaneously generated by the decision maker, or intentionally incorporated into the choice environment (to bias individuals towards some choices and away from others).

Few studies have investigated the influence of partitions on children's decisions. This is in part because many adult studies involve complex instructions or concepts that are not easily accessible to children. One recent study using a child-friendly resource allocation task, broadly similar in structure to previous adult consumer choice and resource allocation tasks, found evidence of partition dependence in children's resource allocations (Reichelson et al., 2019). Children were given 12 "food" tokens to distribute to four different types of zoo animals displayed across three boxes. The boxes were arbitrarily associated with animal types, as the participant observed, to create a three-way box partition and four-way animal partition (two "single animal" boxes each displayed a picture of one animal type, and one "double animal" box displayed a picture of two animal types, see Figure 1). The appearance of the specific animal types across the boxes was counterbalanced, as was the left-right order of single and double boxes.

Equal allocation of food tokens across the animal types would be one possible interpretation of fairness norms implied by the task instructions (the puppet wants to be fair and has food to give to the animals at the zoo). This interpretation would result in equal allocations across box types on average (with participants allocating approximately half of the total food tokens to the two single animal boxes combined, and half to the double animal box). Allocation of food tokens based on an intrinsic preference for a certain animal type would also result in equal allocations across box types on average (again with participants allocating approximately half of the total food tokens to the two single boxes combined, and half to the double animal box). However, an influence of the arbitrary three-way box partition on allocations would result in more food tokens allocated to the two single animal boxes combined than to the double animal box. (A fully partition-based strategy would result in two thirds of allocations going to the single animal boxes, and one third going to the double animal box.) In this task, an influence of the arbitrary three-way box partition, operationalized as more than half of the total number of food 
tokens being allocated to the two single animal boxes combined, is evidence of partition dependence.

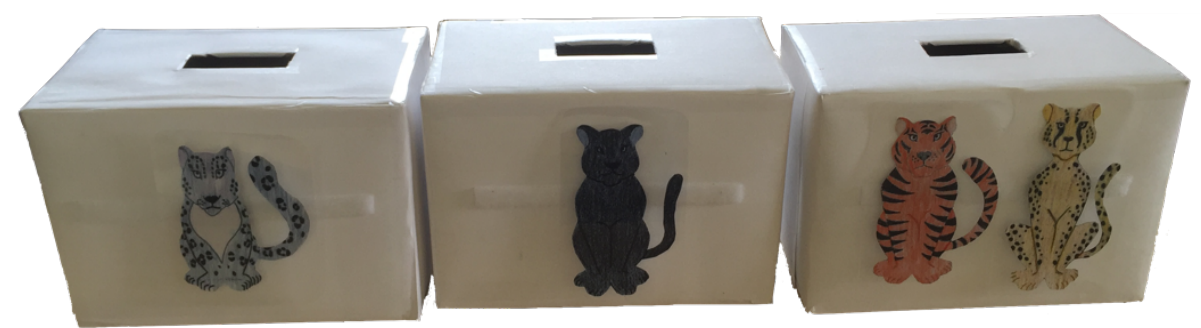

Figure 1. Food token boxes used in the child allocation task (Reichelson et al., 2019). Animals were displayed on the boxes in one of four possible arrangements.

In the study described above (Reichelson et al., 2019), children's hypothetical food allocations were partition dependent: children allocated more than half of the total number of food tokens to the two boxes marked with a single animal type (i.e., the average number of tokens assigned to these two boxes combined was reliably greater than six). In addition, younger children (ages 3 to 6) demonstrated a greater degree of partition dependence than older children (ages 7 to 11), suggesting that naïve decision makers may be more susceptible to partitioning effects, and that these effects may emerge at a very young age rather than developing later with decision making experience (Reichelson et al., 2019). This study provides some of the first evidence that, like adults' financial aid allocations, children's resource distributions are influenced by how the options are partitioned. However, this is the only study showing partition dependence among children, so replication is critical. Further, because the child-friendly task has not yet been conducted with adults, it is not at this point clear whether partition dependent behavior emerges in the same tasks for children and adults.

The first goal of the current work is to broaden our understanding of partition dependence across development by investigating the degree to which children and adults are susceptible to the same partitioning effects. To do this, in Experiment 1 we asked whether adults demonstrate partition dependent behavior on the same resource allocation task previously found to elicit partition dependence in children (Reichelson et al., 2019). The task is to fairly distribute twelve tokens to four types of zoo animals, displayed across three boxes (see Figure 1). If adults, like children, are influenced by the arbitrary three-way box partition, they should allocate more tokens (on average) to the single animal boxes. Specifically, allocating more than half of the total number of tokens (i.e., more than six tokens) to the two single animal boxes combined is evidence of partition dependence in this paradigm.

Although the present task is similar in structure to previous adult allocation tasks (e.g., financial aid allocations to income brackets), we considered the possibility that this particular task might not elicit partition dependence in adults. In addition to the task instructions (the puppet wants to be fair) perhaps suggesting use of particular fairness norms to guide allocation behavior, the multiplicative relations between the individual task items ( 3 boxes, 4 animals, 12 tokens) might be somewhat transparent. Assuming it is relatively easy for adults to recognize that with twelve food tokens, equal (or fair) allocations across the four animal types requires unequal allocations across the three boxes, adults may default to equal allocation across animal types. In fact, it has been suggested that partition dependence is reduced when people have clear criteria for differentiating among individuals and groups, such as when one has expertise in a domain (Fox, Bardolet, et al., 2005). With these issues in mind, we considered that adults may 
not be influenced by the three-way box partition, or might show reduced effects of partition dependence, compared to children, on this child-friendly task.

A second goal was to replicate previous findings of partition dependence in children's resource allocations. To do this, in Experiment 2 we used the same procedure as in Experiment 1, with one modification: participants were given only five food tokens to distribute to the animals. In addition to our goal of investigating the replicability of the effect in children, we were also interested in whether adults would show partition dependence in a version of the task in which it is less clear how to fairly divide five available food tokens among the four animals in three boxes; neither equal allocation to animals nor boxes is possible. We consider the full developmental trajectory of partition dependence including age-related differences and compare the current findings to previous reports of children's performance (Reichelson et al., 2019).

\section{Method}

\section{Experiment 1}

Participants. Eighty adults $\left(M_{\text {age }}=28\right.$ years, range $=18-67$ years; 43 females $)$ participated in the study and were included in subsequent analyses. Eleven of these adults did not provide age information. Three additional adults were excluded for experimenter error.

Procedure. Adults participated in a laboratory room or at a local science museum. The stimuli and task procedure were identical to Reichelson et al. (2019). Participants were shown a gender-neutral puppet, called "Alex", whom they were told had food to distribute to animals at the zoo: "Alex wants to be fair. Can you help Alex give out the food?". Adults were given twelve food tokens (small wooden discs, 2-inch diameter) and were told they could place the tokens in any of the three white boxes to distribute the food to the different animals. The boxes were placed in a row in front of the participant; about $22 \mathrm{~cm} \times 12 \mathrm{~cm} \times 15 \mathrm{~cm}$, with small openings just large enough for the tokens. The two single animal boxes were each marked with a single, distinct animal drawing, and the double animal box was marked with two distinct animal drawings. To convey that the box/animal associations were arbitrary, the researcher assigned individual animal types to the boxes in front of the participant, saying, "Hmmmm, let's put the food for all of the (animal type) at the zoo in this box!" while affixing the animal photos one-byone to the boxes with Velcro. After all the food tokens were allocated, participants were asked which animal they preferred and how many of each animal they thought were at the zoo (e.g., "How many panthers do you think there are at the zoo?").

Stimuli included four drawings of large cats (a leopard, cheetah, panther, and tiger) approximately the same size (about $80 \mathrm{~cm}^{2}$ ). There were two possible physical arrangements of the animal types and box orders, with each animal type appearing an equal number of times on each box type and in each position across a total of four counterbalanced conditions. The two possible physical arrangements of the animal photos were panther and cheetah on single animal boxes and leopard and tiger side-by-side on the double animal box, or the leopard and tiger on the single animal boxes and the panther and cheetah on the double animal box. The two possible box orders were, from left to right: single/single/double and double/single/single. Importantly, the counterbalancing for this task was such that allocations based on an intrinsic preference for a certain animal type would result in equal allocations to single and double boxes, on average. Allocation across animal types would also result in equal allocations across box types, on average. If participants are influenced by the arbitrary three-way box partition, they should allocate more than half of the total number of tokens to the two single animal boxes combined. 
The experimenter recorded the number of tokens allocated to each box and the order in which each token was allocated. After participants distributed the tokens, they were asked which animal they preferred and how many of each animal they thought there were at the zoo. After the study, the experimenter retrieved the tokens from the boxes to confirm the recorded total number of tokens allocated to each box.

\section{Results and Discussion}

We first asked if adults showed partition dependence and found that they did not; adults did not allocate more than half of the total number of tokens to the two single animal boxes combined $(M=6.16, S D=0.80 ; t(79)=1.81, p=.074$, two-tailed, see Figure 2$)$. Only $12.5 \%$ of adults $(n=10)$ allocated seven or more tokens to the two single animal boxes combined and only two adults placed seven or more tokens in one box. Token distribution to single animal boxes did not differ between the single/single/double box order $(M=6.05, S D=0.84)$ and double/single/single box order $(M=6.28, S D=0.76 ; t(78)=-1.31, p=.196)$.

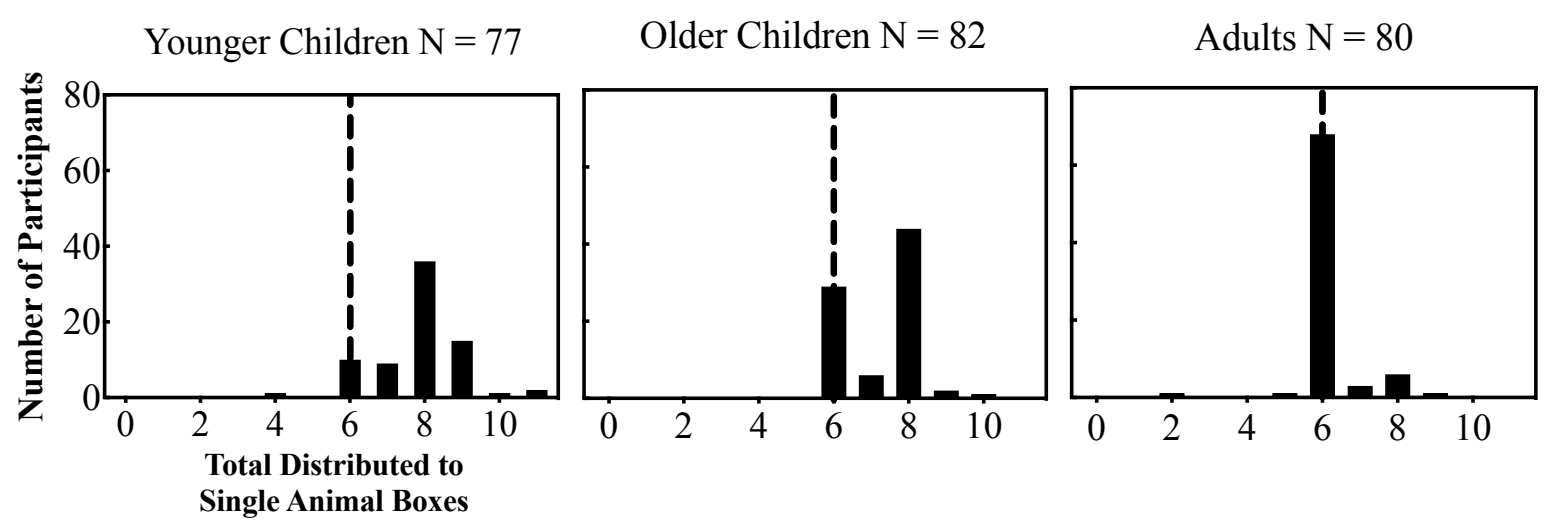

Figure 2. Histograms of the number of tokens allocated to single animal boxes by younger children, ages 3-6 (left), and older children, ages 7-10 (middle) both from Reichelson et al., (2019) and adults' (right) from Experiment 1 here. Evidence of partition dependence in this paradigm consists of allocating more than half (dashed line) of the total number of tokens to the two single animal boxes combined. Consistent with partition dependence, most children allocated more than six tokens to single animal boxes, but some older children and the majority of adults allocated six tokens to single animal boxes.

In Experiment 1, we investigated whether adults showed any evidence of partition dependence in a child-friendly resource allocation task that previously produced partition dependence in children's allocations. Adults' allocations were not influenced by the arbitrary three-way box partition. One possible explanation for this finding is that adults easily recognized that equal allocation to animal types required unequal allocations to box types and so explicitly chose equal allocation to the four animal types. To explore this possibility, in Experiment 2 we reduced the total number of tokens from twelve to five, making neither equal allocation to animals nor boxes possible. We predicted that by making the multiplicative relations between the task elements less transparent, the task would be more challenging for adults and thus more likely to elicit partition dependence in both children and adults. 


\section{Method}

\section{Experiment 2}

Participants. One hundred and eighty-five children $\left(M_{\text {age }}=7\right.$ years and 1 month, range $=3$ years and four months -11 years and 10 months, 104 females $)$ and eighty-seven adults ( $M_{\text {age }}$ $=26$ years, range $=18-64$ years, 47 females) participated in the study. Fifteen children and six adults were excluded due to interference from family members or experimenter error. Remaining participants were one hundred and seventy children $\left(M_{\text {age }}=7\right.$ years and 0 months, range $=3$ years and 3 months -11 years and 8 months, 99 females) and eighty-one adults $\left(M_{\text {age }}=25\right.$ years, range $=18$ years -64 years, 44 females). Three adults did not provide age information but are included in all analyses.

Procedure. The task and procedure were identical to that of Experiment 1, except participants were given only five food tokens to distribute to animals.

\section{Results and Discussion}

We first asked if adults and children showed partition dependence by allocating more than half of the total number of tokens to the two single animal boxes combined. Adults did not do so $(M=2.42, S D=0.57, t(80)=-1.27, p=.207, d=0.01$; see Figure 3$)$ and their allocations to single animal boxes did not differ between the single/single/double box order $(M=2.38, S D=$ $0.54)$ and double/single/single box order $(M=2.46, S D=0.60, t(79)=-0.70, p=.486, d=0.14)$. Children's allocations were consistent with partition dependence. On average, children distributed more than 2.5 tokens to the two single animal boxes combined, $(M=2.90, S D=0.69$, $t(169)=7.52, p<.001, d=0.58$; see Figure 3$)$. No child allocated more than three tokens to a single box indicating that these results could not have been driven by allocation patterns inconsistent with diversification, such as putting all five tokens in one box. Like adults, children's allocations to single animal boxes did not differ between the single/single/double box order $(M=2.93, S D=0.64)$ and double/single/single box order $(M=2.87, S D=0.75) ; t(168)=$ $0.53, p=.597$ ). These findings demonstrate that children (all ages), but not adults, were influenced by the arbitrary three-way box partition.

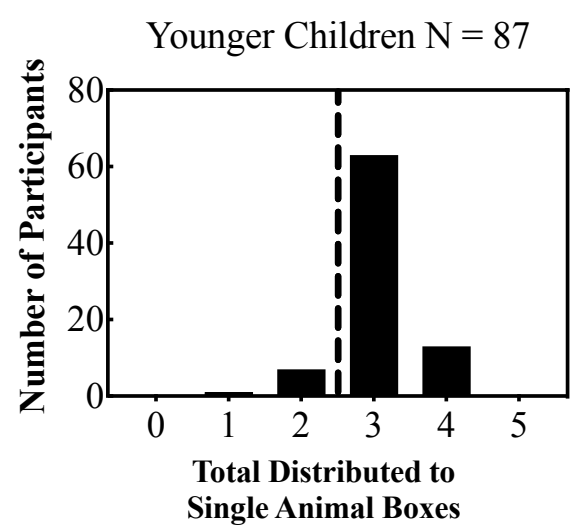

Older Children $\mathrm{N}=83$

Adults $\mathrm{N}=81$
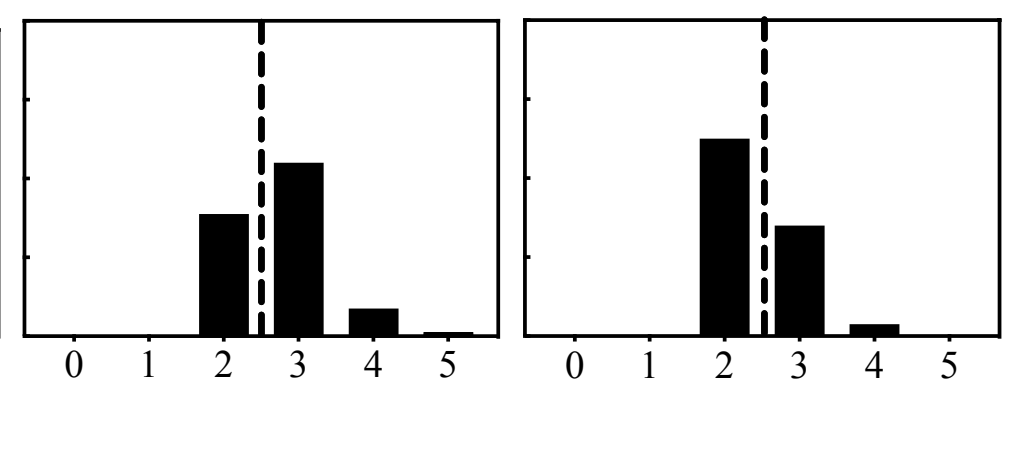

Figure 3. Histograms of the number tokens allocated to single animal boxes by younger children, ages 3-6 (left), older children, ages 7-10 (middle) and adults (right) in Experiment 2. Evidence of partition dependence consists of a bias toward allocating more than half (dashed line) of the total number of tokens to the two single animal boxes combined. Consistent with partition dependence, most children allocated more than 2.5 tokens to single animal boxes. Some older children and the majority of adult participants did not allocate more than 2.5 tokens to single animal boxes. 
Consistent with previous findings with children on a similar 12-item allocation task (Reichelson et al., 2019), there was a significant negative correlation between number of tokens distributed to the two single animal boxes and age in months, suggesting that younger children in our study were more susceptible to partition dependence $(r(167)=-0.28, p<.001)$ (see Figure 4). Following Reichelson et al. (2019), we split children into two groups: "younger" children ages 3 to $6\left(n=87 ; M_{\text {age }}=5\right.$ years and 2 months, range $=3$ years and 3 months -6 years and 7 months, 46 females $)$ and "older" children ages 7 to $11\left(n=83 ; M_{\text {age }}=8\right.$ years and 8 months, range $=7$ years and 0 months -11 years and 4 months, 53 females). Children in both age groups distributed more than half of the total number of tokens to single animal boxes combined (older: $M=2.73, S D=0.66, t(82)=3.22, p=.002, d=0.35$; younger: $M=3.06, S D=0.69, t(86)=$ $7.56, p<.001, d=0.81)$. However, younger children distributed significantly more tokens to single animal boxes compared to older children $(t(168)=3.11, p=.002, d=0.49)$; $90 \%$ of younger children and $63 \%$ of older children distributed tokens in a way that was consistent with partition dependence. Token distribution did not differ between the single/single/double box order (older: $M=2.83, S D=0.59$; younger: $M=3.02, S D=0.66$ ) and double/single/single box order (older: $M=2.65, S D=0.72$; younger: $M=3.09, S D=0.72$ ); all $p$ s $>.200$.

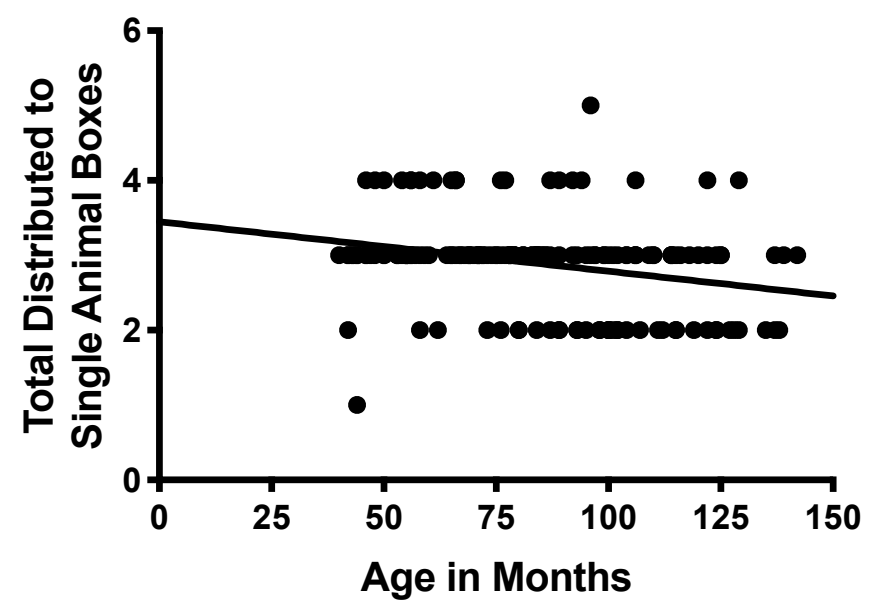

Figure 4. A scatter plot of total number of tokens distributed to the two single animal boxes (combined) as a function of age.

The goals of Experiment 2 were to investigate whether adults demonstrate partition dependence in a 5-item allocation task and to replicate previous findings of partition dependence in children. Consistent with past work, children exhibited strong partitioning effects, and younger children appeared more susceptible to the effects of partition dependence than older children. Adults' allocations were consistently biased towards equal allocations across animal types and were not influenced by the arbitrary three-way box partition.

\section{General Discussion}

To investigate the developmental trajectory of partition dependence, in two experiments we asked whether children and adults are similarly influenced by partition dependence in a childfriendly resource allocation task: distributing hypothetical food tokens to four types of animals, displayed across three boxes. The task does not require equal allocations to boxes or animals, but counterbalancing was such that equal allocation of tokens across the four-way animal partition, 
or allocations based on an intrinsic preference for a certain animal type, would lead to equal allocations across box types on average. An allocation pattern based on the arbitrary three-way box partition (allocating more than half of the total number of tokens to the single animal boxes combined, on average) is evidence of partition dependence.

Experiment 1 used a 12-item resource allocation task previously found to elicit partition dependent behavior in children (Reichelson et al., 2019) to investigate whether the task would elicit similar behavior in adults; it did not. In Experiment 2 we investigated whether children and adults show similar patterns of partition dependence when the number of available resources is reduced from twelve to five items. Critically, this makes the multiplicative relations between the task elements less transparent: five resources cannot easily be divided across four animals or three boxes. We predicted that when the multiplicative relations between the task elements are more challenging to represent, adults may rely on both fairness norms and arbitrary box-based partitions for allocation of resources and thus may be more likely to show partition dependence. Still, children but not adults showed robust partitioning effects.

The finding here of robust partition dependence in children's resource allocations replicates and extends the work of Reichelson et al. (2019), providing additional evidence that partitioning may influence decisions from a very young age, rather than after years of decisionmaking experience. In contrast, the current findings with adults are inconsistent with the literature: previous studies have found evidence of robust partition dependence in adults' resource allocations using different tasks (e.g., financial aid allocations; Fox, Bardolet, et al., 2005; Xing et al., in revision). Why do children, but not adults, exhibit partition dependence in the resource allocation task used here? We outline three possible explanations below.

One possibility is that children and adults differ in how they interpret the arbitrary partitions they observe. In the present task, the association of each animal type with its box was arbitrarily generated in front of the participant instead of being presented as a pre-established partitioning of options (as in previous financial aid tasks) to communicate that, at least in this task context, the three-way box partition is arbitrary. If the act of associating animals with boxes in front of the participant sufficiently communicated that the three-way box partition is arbitrary, participants should attend to the four-way animal partition. On the other hand, if children see the experimenter as an authority who intentionally groups the animals by box type - thus making the three-way box partition appear less arbitrary and more salient - children may have been more likely than adults to interpret the box partition as meaningful. Interestingly, adults who show partition dependence have also been found interpret arbitrary partitions as conveying contextual meaning (e.g., about the relative popularity of options; Tannenbaum, Fox, \& Goldstein., working paper), similar to inferences children may make.

A second possibility is that children and adults differ in how they interpret the task instructions to be fair and to give food to the animals. Adults may have been more likely to interpret these instructions as indicating a strict animal-based equal allocation strategy. Children, in contrast, may have been less certain of how to interpret the instructions and, as a result, more likely to rely on a naïve diversification strategy. Such a pattern would be consistent with studies showing that, even among adults, novices in a domain show greater partition dependence than experts (e.g., Fox, Ratner et al., 2005), which may be due to experts having clearer criteria for allocating resources (Fox, Bardolet, et al., 2005). We note that we do not, however, think the findings result from an inability of children to share fairly more generally. Many children in our study allocated tokens one at a time to each box, suggesting a strategy of matching tokens to boxes or animals via one-to-one correspondence. Furthermore, even before preschoolers can 
count, they understand norms of equal sharing and are able to use turn-taking strategies, demonstrate awareness of the equality norm for dividing resources, and fairly distribute resources (e.g., Chernyak, Harris, \& Cordes, 2019).

A third possibility is that even though we increased the complexity of the multiplicative relations in Experiment 2, the relationship among tokens, animals, and boxes may have remained transparent to adults. Adults may have had a clearer understanding that equal division of tokens across animals requires unequal division across boxes and may have explicitly chosen to diversify over animals even though this would mean unequal assignment to boxes. This explanation seems somewhat less likely than other possibilities, however, it is worth considering here given that adults routinely show partition dependence in situations similar in quantitative complexity to those of the present Experiment 2. For example, when asked to allocate four meals to three time periods over the course of an academic year, students allocated a greater number of meals to whichever semester was further partitioned into two quarters (Fox, Ratner, et al., 2005). As we indicated earlier, explicitly dividing tokens over animals is not necessary in the present task in order to allocate tokens equally across animals. However, it is possible that the use of turn taking by children without any a priori allocation plan (e.g., give three tokens to each animal) might lead to more points of uncertainty during the task, increasing the opportunity for susceptibility to biases.

Rather than reflecting new potential contributors to partition dependence, what these possibilities suggest is that children and adults may be subject to the same types of influences on resource allocation behavior. Children may show partition dependence across a greater number of tasks than adults precisely because children are less likely to have domain expertise, more likely to attribute meaning to the arbitrary partitions presented by adults, and less skilled with complex divisions of resources across items and groups. If this is the case, we should generally expect to see a trajectory of decreasing partition dependence across childhood, as in the present studies, with partition dependent behavior observed in adults only for more difficult tasks. A promising direction for future work is further consideration of how people's conceptualizations of resource allocation tasks change across the lifespan (along the dimensions outlined here; see Fox, Bardolet, et al., 2005, for review) and how these changes in conceptualization are related partition dependent behavior.

In sum, the current work contributes to our understanding of the extent to which children's everyday decisions are subject to partitioning effects beginning from a very young age. Past research with adults has shown that some individuals are more heavily influenced by partitioning effects on certain tasks, and the findings presented here suggest that there are also age-related differences at least in the context tested here: children's resource allocations were heavily influenced by partitioning effects while adults did not show any signs of partition dependence. Additionally, younger children appeared more susceptible to the effects of partition dependence than older children. A better understanding of partition dependence throughout development, as well as its underlying mechanisms, may be used to help parents and teachers promote optimal cognitive, physical, and social growth by nudging young children to make better decisions regarding their engagement with certain toys or activities. Older children are also faced with decisions that could have long-term consequences for school success, socialization, and health and nutrition (e.g., decisions about extracurricular activities or subjects in school).

Future research will need to consider the many ways in which decision makers of all ages may be influenced by how options are grouped to better understand how both adults' and children's decisions are uniquely influenced by the subjective partitioning of available options, 
the conditions under which partition dependence is likely to appear, and any possibility of mitigating its negative effects. Knowledge of this strong systematic effect may be influential in empowering individual decision makers and encouraging them to make a conscious effort to reduce the effects of partition dependence in their decisions while considering the possible ways to diversify over a particular dimension. 


\section{References}

Benartzi, S., \& Thaler, R. (2001). Naive Diversification Strategies in Defined Contribution Saving Plans. The American Economic Review, 91, 79-98.

Chernyak, N., Harris, P. L., \& Cordes, S. (2019). Explaining early moral hypocrisy: Numerical cognition promotes equal sharing behavior in preschool-aged children. Developmental Science, 22, 1-13. https://doi.org/10.1111/desc.12695

Fox, C. R., Bardolet, D., \& Lieb, D. (2005). Partition Dependence in Decision Analysis, Resource Allocation, and Consumer Choice. Experimental Business Research, 229-251. https://doi.org/10.1007/0-387-24244-9_10

Fox, C. R., Ratner, R. K., \& Lieb, D. S. (2005). How subjective grouping of options influences choice and allocation: Diversification bias and the phenomenon of partition dependence. Journal of Experimental Psychology: General, 134, 538-551. https://doi.org/10.1037/00963445.134.4.538

Fox, C. R., \& Rottenstreich, Y. (2003). Research Article. Psychological Science, 14, 195-200. https://doi.org/10.23959/sfnrl-1000005

Read, D., Antonides, G., Van den Ouden, L., \& Trienekens, H. (2001). Which Is Better: Simultaneous or Sequential Choice? Organizational Behavior and Human Decision Processes, 84, 54-70. https://doi.org/10.1006/obhd.2000.2917

Read, D., \& Loewenstein, G. (1995). Diversification Bias: Explaining the Discrepancy in Variety Seeking Between Combined and Separated Choices. Journal of Experimental Psychology: Applied, 1, 34-49. https://doi.org/10.1037/1076-898X.1.1.34

Reichelson, S., Zax, A., Patalano, A. L., \& Barth, H. C. (2019). Partition dependence in development: Are children's decisions shaped by the arbitrary grouping of options? Quarterly Journal of Experimental Psychology, 72, 1029-1036. https://doi.org/10.1177/1747021818777720

Simonson, I. (1990). The Effect of Purchase Quantity and Timing on Variety-Seeking Behavior. Journal of Marketing Research, 27, 150. https://doi.org/10.2307/3172842

Tannenbaum, D., Doctor, J. N., Persell, S. D., Friedberg, M. W., Meeker, D., Friesema, E. M., ... Fox, C. R. (2015). Nudging Physician Prescription Decisions by Partitioning the Order Set: Results of a Vignette-Based Study. Journal of General Internal Medicine, 30, 298-304. https://doi.org/10.1007/s11606-014-3051-2

Tannenbaum, D., Fox, C. R., \& Goldstein., N. J. (working paper). Partitioning menu items to nudge single-item choice.

Xing, C., Williams, K., Hom, J., Kandlur, M., Owoyemi, P., Peters, R., ... Barth, H. (2020). Partition dependence in financial aid distribution to income categories. Revised manuscript under review. 